\title{
APLICACIÓN DE LOS SISTEMAS DE INFORMACIÓN GEOGRAFICA PARA LA GESTIÓN DE LA MALLA VIAL DE LA CIUDAD DE MEDELLÍN
}

\author{
Jaime A. Zapata Duque \\ Alcaldía de Medellín, Colombia \\ jaimea.zapata@medellin.gov.co
}

\author{
Gabriel J. Cardona Londoño \\ Alcaldía de Medellín, Colombia \\ gabriel.cardona@medellin.gov.co
}

(Tipo de Artículo: Investigación. Recibido el 04/12/2012. Aprobado el 30/12/2012)

\begin{abstract}
RESUMEN
El estado de la malla vial depende fundamentalmente de factores tales como el tráfico, características estructurales y funcionales del pavimento y condiciones climatológicas locales, y su mantenimiento tiene importantes implicaciones espaciales. Este documento describe el proceso de implementación del sistema de gestión de pavimentos de la ciudad de Medellín, desarrollado en una plataforma SIG. El sistema está soportado en una geodatabase corporativa de inventario y diagnóstico, el cual alimenta un software de modelación del deterioro del pavimento y arroja recomendaciones de intervención en cada segmento vial. Este sistema sirve como apoyo a las autoridades encargadas a la toma de decisiones, con el fin de realizar la planeación del mantenimiento de la malla vial, optimizar los recursos disponibles y ofrecer un mejor servicio a la comunidad.
\end{abstract}

\section{Palabras clave}

Sistemas de información geográfica (SIG), Transporte, vehículos, transporte por carretera, mantenimiento, asfalto, áreas urbanas, gestión.

\section{APPL YING GEOGRAPHIC INFORMATION SYSTEMS FOR THE MANAGEMENT OF THE ROAD NETWORK OF MEDELLÍN CITY}

\begin{abstract}
The road network condition depends mainly on factors such as traffic, structural and functional characteristics of asphalt and local weather conditions, and its maintenance, have important spatial implications. This document describes the implementation process of an asphalt management system for Medellin city, developed in a GIS platform. The system is supported in an inventory and diagnostic geodatabase, which feeds the modeling software for asphalt deterioration, and provides intervention recommendations for each road segment. This implementation helps supporting the decision making process for authorities, in order to perform the planning of road network maintenance, optimizing available resources and providing better service to community.
\end{abstract}

\section{Keywords}

Geographic Information Systems (GIS), Transportation, Vehicles, Road transportation, Maintenance, Asphalt, Urban areas, Management

\section{APPLICATION DES SYSTEMMES D'INFORMATION GEOGRAPHIQUE POUR LA GESTION DU RESEAU ROUTIER DE LA VILLE DE MEDELLÍN}

\begin{abstract}
Résumé
La condition du réseau routier est déterminée par des facteurs comme la circulation, de caractéristiques structurales et fonctionnelles de l'asphalte et des conditions climatologiques locales, et sa maintenance a dans implications spatiaux significatifs. Cet article décris le processus d'implémentation du système de gestion d'asphaltes de la ville de Medellín, développé sur une plateforme SIG. Le système est supporté sur une geo-base de données corporatif d'inventaire et diagnostic, qui supporte un logiciel de modélisation de la détérioration de l'asphalte et nous donne des recommandations pour l'intervention sur chaque segment routier. Ce système supporte aux autorités responsables pour la prise des décisions, avec le but de réaliser la maintenance du réseau routier, d'optimiser les ressources disponibles et d'offrir un meilleur service aux citoyens.
\end{abstract}

\section{Mots-clés}

Système d'information Géographique (SIG), transport, véhicule, Maintenance, transport routier, asphalte, gestion. 


\section{INTRODUCCIÓN}

La malla vial es el componente principal del sistema de transporte terrestre sobre la cual se fundamenta la movilidad de bienes y personas dentro de una entidad territorial. El mantenimiento de la infraestructura vial es una prioridad para las autoridades encargadas de su administración, ya que representa un patrimonio muy valioso para la economía de la región en la que se encuentra. La adecuada administración de la malla vial conlleva a realizar la planeación y el mantenimiento de su infraestructura en óptimas condiciones, mediante la identificación de las necesidades, la priorización de las intervenciones y la optimización de los recursos.

Una malla vial en buen estado contribuye a la articulación entre los puntos de origen y destino de los diferentes viajes que se generan, lo cual se traduce en una mayor competitividad y desarrollo del transporte e infraestructura del territorio del cual hace parte. Esto se ve reflejado en la realización de viajes más rápidos, cómodos, seguros y económicos.

Los desplazamientos que a diario se realizan sobre la infraestructura vial, tanto en modos de transporte individuales como masivos y de carga, ocasionan un deterioro progresivo sobre el pavimento. Así mismo, las características geomecánicas de la estructura del pavimento, las condiciones ambientales, geológicas, los drenajes y las condiciones del suelo, contribuyen significativamente al detrimento de la malla vial, reflejándose en la aparición de fallas superficiales e irregularidad en la carpeta asfáltica.

El deterioro de la malla vial tiene repercusiones económicas, ambientales, sociales y políticas, dentro de las cuales se pueden mencionar, desde el punto de vista de los usuarios, el aumento en los costos de operación vehicular, en tiempos de desplazamiento, inconformidad, accidentalidad, congestión y consumo de combustible; desde el punto de vista de la infraestructura, el incremento en las intervenciones sobre la capa de rodadura y la estructura del pavimento; y desde el punto de vista ambiental con el aumento de los niveles de contaminación del aire y por ruido, en resumen una pérdida de confianza y credibilidad en los gobiernos responsables.

Todos estos factores pueden ser caracterizados, analizados e integrados espacialmente a través de los Sistemas de Información Geográfica (SIG). Esta herramienta de alto poder de análisis, interpretación y visualización, que emplea tecnología de avanzada mediante hardware y software especializados, permite integrar datos alfanuméricos con la información geográfica. Los SIG realizan dicha integración mediante el despliegue de diferentes capas temáticas de estudio, tales como la red vial, topografía, usos del suelo, red hídrica, catastro, equipamientos colectivos, espacios públicos, división administrativa, sistemas de transporte, obras de infraestructura, entre otras. De esta manera, es posible realizar procesos complejos de análisis multicriterio con el fin de tomar decisiones y optimizar los recursos en la solución de un problema en particular.
Dentro del ámbito de aplicación de los SIG, la red vial de un territorio se representa mediante líneas segmentadas, conectadas entre sí, con integralidad, consistencia y continuidad, que permiten clasificar, visualizar, interpretar y analizar los atributos inherentes a la calzada vehicular. De esta manera se representan diferentes capas temáticas, tales como tipos de superficie de rodadura, estados de condición, jerarquía vial, tipo de tráfico, pendientes, tipos de intervención; en general, un conjunto de información que permite al usuario de los SIG disponer de un mejor entorno visual, que le facilita el análisis de la información.

\section{ESTADO DEL ARTE}

Existen algunas investigaciones previas en el marco de la implementación de los SIG en los temas relacionados con vías. A continuación mostraremos algunos de estos importantes antecedentes.

P. Álvarez y otros [1] desarrollaron un modelo de planificación del mantenimiento de la red de carreteras en Badajoz, España, el cual permite determinar las condiciones de las vías, evaluar sus indicadores y llevarlos a un ambiente SIG, con el fin de visualizar su distribución según el estado, con esta información se pueden realizar diagnósticos y optimizar los recursos asignados. Los factores que afectan el mantenimiento de las vías, tales como el tráfico, estado superficial y calidad de la señalización, permiten medir el grado de satisfacción de los usuarios y definir los sitios de intervención. Mediante la visualización geográfica, se observan las condiciones más adecuadas de las vías para la seguridad de los usuarios, la preservación de las estructuras viales, la pavimentación de las vías, la señalización horizontal y vertical y los taludes. Los SIG ofrecen numerosas aplicaciones y análisis de la información, apoyando la toma de decisiones sobre la inversión de los recursos estatales de manera objetiva y técnica.

Por otro lado J. Coutinho [2], considera varios criterios de evaluación y alternativas en la planificación y modelamiento de un territorio, dada la importancia que tienen los aspectos sociales, económicos, ambientales y técnicos, en la toma de decisiones, todo esto para ofrecer a los usuarios un entorno flexible y fácil de usar en la planificación de la infraestructura urbana. En la evaluación de las inversiones se consideran criterios múltiples y aplicaciones espaciales en la distribución de costos y beneficios, utilizando métodos de análisis como el DSS (Decisión Support Systems), facilitando la relación entre la toma de decisiones y los modelos computarizados.

W. Chen y otros [3] plantean que el propósito de un sistema de gestión de pavimentos es determinar la intervención más efectiva de mantenimiento y reconstrucción en un territorio, integrando tecnología SIG y GPS para la recolección y almacenamiento de datos, análisis geoespacial y optimización de la planeación del mantenimiento, con lo cual se aumenta la capacidad y utilidad del modelo de gestión. En el caso del aeropuerto 
de Shanghai se crearon bases de datos espaciales mediante la segmentación del pavimento y la identificación década tramo con un único código, con lo cual se generó un mapa y se recolectaron los datos con GPS permitiendo de esta manera disminuir el trabajo y mejorar la productividad. Adicionalmente se utilizó una tecnología geoespacial mediante cartografía de mapas temáticos con información de la condición del pavimento y se identificaron las fallas en las losas para determinar las medidas correctivas. Así los SIG permitieron realizar el análisis y ejecución de una gestión óptima del mantenimiento del pavimento, con lo cual se logró mejorar la toma de decisiones y resolver problemas a futuro.

En el trazado de carreteras en Beirut, Líbano, se utilizó un enfoque de evaluación integrado empleando criterios generados bajo una plataforma SIG, con la cual se evaluaron las alternativas para el alineamiento de las vías. Además se tuvieron en cuenta consideraciones geotécnicas y medio ambientales para la realización de modelos del diseño de las autopistas, la estabilidad de las vertientes y el ruido del tráfico. Con este trabajo se logró mostrar el potencial del enfoque desarrollado, que permite incorporar nuevos criterios en el diseño del trazado de carreteras y automatizar el procedimiento de evaluación.

En el desarrollo de un prototipo de análisis de impacto del tráfico se pueden integrar los SIG para generar modelos de simulación y visualización en 3D. De esta manera

Wang [4] empleó SIG con distintos modelos para proyectar el tráfico, la velocidad vehicular y las concentraciones de monóxido de carbono. Apoyados en modelos en $3 \mathrm{D}$ de los segmentos viales y edificios a lo largo de la carretera los autores realizaron una presentación en 3D con experiencias de conducción, lo que brinda amplias posibilidades de toma de decisiones a los planeadores en los proyectos de investigación y práctica [4].

Durante las últimas décadas, se ha extendido la aplicación del SIG en diversos temas de la Ingeniería de Infraestructura Vial, con aplicación en sistemas de administración de la red vial. A nivel nacional cabe mencionar lo realizado en Medellín, Bogotá y Cali, a nivel latinoamericano los países que cuentan con esta aplicación son Argentina, Brasil, Panamá y Perú, y a nivel internacional Israel, EEUU y España, entre otros.

La empresa de consultoría TNM Ltd., ha desarrollado a nivel mundial varias aplicaciones de los sistemas de gestión vial. En Israel se evaluaron las características de los pavimentos para alimentar la base de datos del sistema de la red interurbana [5]. En la ciudad de Panamá, se efectuó la actualización del inventario de la red vial interurbana $(8.500 \mathrm{Km}$ de vías pavimentadas y no pavimentadas y 1384 puentes) y se hicieron estudios de tráfico. En Perú se realizó el inventario de la red vial nacional pavimentada (aprox. $8.500 \mathrm{Km}$ ) y los puentes del país y se implementó el sistema de valoración de la red nacional. En la ciudad de Bogotá se realizó la actualización del inventario y diagnóstico de la malla vial urbana (232.7 Km-calzada), nuevas vías urbanas (70.3 $\mathrm{Km}$-calzada) y nuevas vías rurales [5]. Y, en el ámbito regional, se puede mencionar que a partir del año 2005 se llevó a cabo el inventario y diagnóstico de la malla vial urbana y rural de la ciudad de Medellín en una longitud de $2013 \mathrm{Km}$-calzada.

Es importante tomar en cuenta el caso en la ciudad de Los Ángeles, USA, en el año 2008 el Departamento de Obras Públicas donde se realizó la evaluación de la infraestructura vial, con el fin de mejorar dicha infraestructura. Para este efecto se utiliza un sistema de alta tecnología que permite determinar el mantenimiento - la rehabilitación requerida, predecir las condiciones futuras del pavimento y establecer el tiempo óptimo para ejecutar dichas tareas. Para cada condición se utilizó el "Indice de Condición del Pavimento" (PCI), el cual evalúa las condiciones físicas de las vías según las características estructurales y operacionales basado en la metodología Micro PAVER que determina el tipo, la severidad y la cantidad de fallas superficiales [6].

La ciudad de Medellín, por su parte, presenta unas características particulares que influyen específicamente en el comportamiento de los pavimentos; factores, tales como la geomorfología, geografía, medio ambiente, drenajes, demografía, usos del suelo y división política administrativa, entre otros. Por esta razón, es indispensable la implementación de un sistema de gestión vial acorde a las características propias de la ciudad y desarrollado sobre la plataforma SIG.

La implementación del sistema de gestión vial en la ciudad de Medellín tiene como fin primordial realizarla planeación del mantenimiento de la malla vial y su infraestructura asociada. El sistema está soportado en una geodatabase corporativa de inventario y diagnóstico, con la cual se alimenta un software que realiza la modelación del deterioro del pavimento a lo largo del tiempo. Este software está basado en el modelo HDM-4 (Metodología del Banco Mundial) y fue calibrado para las condiciones particulares de la ciudad, con el fin de obtener resultados reales, que respondan a las características de la ciudad y de la red vial.

Luego de este proceso, el sistema de gestión vial arroja diferentes recomendaciones de intervención para cada segmento vial seleccionado, de acuerdo con una matriz de decisiones que fue diseñada según las especificaciones propias de la Secretaría de Infraestructura Física de la Alcaldía de Medellín. Esta matriz de decisiones involucra tres parámetros principales con los cuales procesa los datos: el tráfico, la capacidad estructural y las condiciones superficiales y de servicio del pavimento. De esta manera, el sistema de gestión vial permite identificar las necesidades y priorizar las intervenciones de acuerdo con varios criterios de selección tales como: el tipo de intervención, la jerarquía vial, la relación costo-beneficio o el menor costo. Finalmente, el resultado de esta implementación logra apoyar la toma de decisiones por parte de las 
autoridades encargadas, y así realizar la planeación del mantenimiento de la malla vial, optimizar los recursos disponibles y ofrecer un mejor servicio a la comunidad.

\section{MARCO TEÓRICO}

El primer elemento a considerar en la implementación del Sistema de Gestión Vial, es la recopilación de los datos de campo, es decir la elaboración del Inventario y Diagnóstico del estado actual de la malla vial de la ciudad de Medellín, a través de la obtención de los parámetros geométricos y técnicos. El segundo elemento es la elaboración de una Matriz de Decisiones, cuyo objetivo básico consiste en establecer la intervención requerida en un segmento vial para mantener un nivel de servicio adecuado y proteger el patrimonio vial en un cierto período de tiempo.

\subsection{INVENTARIO Y DIAGNÓSTICO DE LA MALLA VIAL}

Los parámetros geométricos y técnicos incluyen el inventario de fallas superficiales en el pavimento MDR, medida del coeficiente de resistencia al deslizamiento - CRD, mediciones de deflectometría, medida del índice de regularidad superficial - IRI, determinación de espesores de la estructura de pavimento y conteos de tránsito para la asignación del tránsito promedio diario -TPD, los cuales serán posteriormente insumos para el modelo de priorización de los segmentos viales. Un segmento vial se define como la porción de vía entre dos intersecciones, que tiene un nodo inicial y final, una dirección y sentido.

El segmento vial es el elemento geográfico básico de visualización, de tipo lineal conectado entre dos nodos, el cual se identifica mediante un identificador de segmento (ID_OP) compuesto por un código de siete (7) dígitos. El primer número del código representa la jerarquía vial de acuerdo al Plan de Ordenamiento Territorial - POT de la ciudad, los otros seis (6) dígitos corresponden a un consecutivo de asignación para los segmentos clasificados dentro de cada jerarquía vial. De esta manera, el (1) representa las autopistas urbanas, el (2) las arterias principales, el (3) las arterias menores, el (4) las vías colectoras, el (5) las vías de servicio, el (6) las vías rurales cabecera y el (7) las vías rurales veredales.

La determinación de los parámetros técnicos mencionados anteriormente, se realiza con equipos de alto rendimiento, logrando una mayor captura de datos, mayor eficiencia, disminución de costos y menor impacto ambiental. La definición y forma de medir cada parámetro se describe a continuación.

\subsubsection{Parámetros Técnicos del Pavimento}

Los parámetros técnicos del pavimento describen las diferentes características de cada una de las capas que lo componen. Estas capas pueden ser variables y van desde la superficie de rodadura hasta la subrasante o suelo de apoyo de la estructura, pasando por las capas intermedias o material granular. Los principales parámetros técnicos que se analizaron son el índice de rugosidad internacional, las deflexiones, los espesores de las capas, el coeficiente de resistencia al deslizamiento, la capacidad de soporte de la subrasante y el índice de condición global del pavimento, las cuales se describen a continuación.

\subsection{2. Índice de Regularidad Internacional- IRI}

La regularidad superficial, expresada normalmente por un índice, se define como las deformaciones verticales acumuladas a lo largo de un kilómetro con respecto a un plano horizontal en un pavimento, denominadas irregularidades. Estas deformaciones se deben al procedimiento constructivo y al efecto producido sobre la vía por el tránsito vehicular o por la combinación de ambas. El IRI se mide con el equipo denominado perfilógrafo láser RSP de medición directa, sin contacto con la vía, de clase II según la FHWA (Federal Highway Administration), y se obtiene midiendo el perfil longitudinal [7].

La superficie de rodadura del pavimento proporciona al usuario características de confort, seguridad y durabilidad, lo cual se logra a partir del cumplimiento de ciertos parámetros. La superficie de rodadura de un pavimento se puede clasificar conforme a las irregularidades superficiales presentes. EI IRI es una característica del pavimento flexible importante, ya que afecta la calidad del desplazamiento, los costos de operación y el mantenimiento de los vehículos.

Con el fin de estandarizar el valor de la regularidad superficial, el Banco Mundial propuso el Índice Internacional de Regularidad (IRI), que se basa en un modelo matemático denominado cuarto de carro normalizado (Golden Quarter Car) circulando a 80 $\mathrm{km} / \mathrm{h}$. Dicho índice se obtiene a partir de la acumulación del desplazamiento relativo entre las masas de la carrocería y la suspensión del modelo, cuando el vehículo circula por el perfil de la vía en estudio. Entre más alto es el valor del IRI, más mala se considera la condición superficial.

\subsubsection{Deflectometría}

La deflexión es una medida de deformación del pavimento flexible, representa una respuesta total del sistema o estructura del pavimento ante la aplicación de una carga externa. La condición estructural de un pavimento se evalúa midiendo la deflexión vertical que presente éste bajo una carga normalizada. La medición se realiza en forma no destructiva y se utiliza para relacionarla con la capacidad estructural del pavimento. Es importante destacar que no sólo se desplaza el punto bajo la carga, sino también en un sector alrededor de ella, causando un conjunto de deflexiones, el cual se denomina cuenco o bulbo de deflexiones como se observa en la Figura 1.

El equipo empleado para la medición de deflexiones es el Deflectómetro de Impacto (Falling Weight Deflectometer - FWD). El principio general de funcionamiento de este equipo de alto rendimiento se basa en la producción de una onda de carga sobre el pavimento, lo cual se consigue mediante el impacto generado por la caída libre de una masa, que es 
transmitido mediante un sistema de amortiguamiento elástico montado sobre una placa de carga. Los sensores de deflexión utilizados tienen una serie de siete geófonos para registrar las velocidades y los desplazamientos (D0, D20, D60, D90, D120, D150 y D180), donde D0 es la deflexión medida bajo el punto de aplicación de la carga y D180 es la deflexión medida en el punto más alejado de la carga [8].
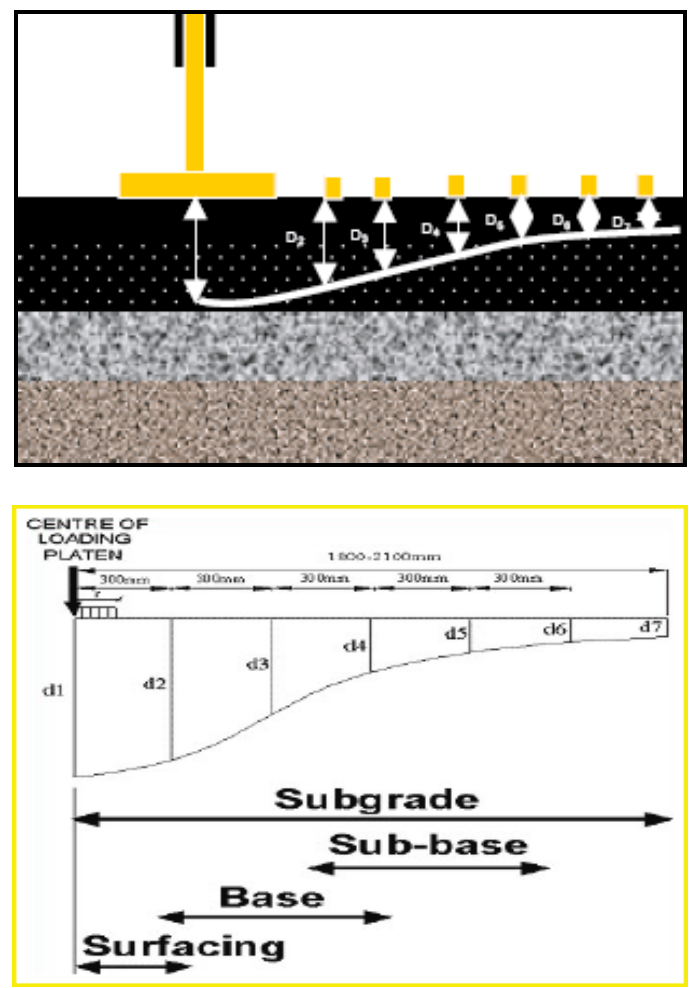

Fig. 1. Bulbo de deflexiones [8]

\subsubsection{Espesores del Pavimento}

La determinación de espesores de la estructura del pavimento, permite, conjuntamente con la deflectometría, analizar la capacidad estructural del pavimento. Es así como el número estructural - SN en pavimentos flexibles, depende directamente de la medida de la deflexión y de los espesores de la estructura del pavimento y permite establecer la condición estructural del mismo.

La información de espesores del pavimento para los segmentos viales se recopiló mediante la metodología Ground Penetrating Radar (GPR), la cual emplea un dispositivo que emite un pulso corto de energía electromagnética, capaz de determinar la presencia o ausencia de un objeto mediante el examen de la energía reflejada de dicho pulso. El equipo utilizado fue el Georadar, el cual sondea el subsuelo por medio de impulsos electromagnéticos de alta frecuencia. En una estructura de pavimento la onda electromagnética viaja hasta que se encuentra con una discontinuidad dieléctrica debida a un cambio de material, como una nueva capa del pavimento, humedad, presencia de cámaras de aire o cualquier otro fenómeno por el que cambie la constante dieléctrica del material. Una parte de la onda es reflejada por esta discontinuidad y el resto continúa su camino hacia el interior del pavimento. Controlando con exactitud el tiempo de viaje de la onda desde su inicio hasta la recepción de la onda reflejada, es posible la determinación de los espesores de cada capa de pavimento [9].

\subsubsection{Coeficiente de Resistencia al Deslizamiento - CRD}

La resistencia al deslizamiento es la fuerza desarrollada entre la superficie del pavimento y los neumáticos de los vehículos. El coeficiente de fricción del pavimento, depende entre otras cosas, del tipo de pavimento, el estado superficial y las condiciones de humedad. EI CRD se puede determinar en cada una de las calzadas vehiculares empleando el péndulo inglés, ensayo normado en el INV-E-792, o el Grip Tester, el cual es un equipo de alto rendimiento [10].

\subsubsection{California Bearing Ratio - CBR}

EI CBR está definido como la fuerza requerida para que un pistón normalizado penetre a una profundidad determinada. Está expresado en el porcentaje de fuerza necesaria para que el pistón penetre a esa misma profundidad y con igual velocidad, en una probeta normalizada constituida por una muestra patrón de material remoldeado. El CBR se determina en la capa de la sub-rasantea través de apiques, del suelo que está por debajo de la estructura del pavimento. La expresión que define al CBR, es la siguiente [11]:

$$
C B R=292 / P D C^{1.12}
$$

Donde el PDC (Penetrómetro dinámico de cono) portátil, constituido por una masa deslizante que se deja caer por gravedad sobre un yunque de impacto, y que por medio de una varilla transmite la energía a una puntaza cónica que se hinca en el terreno. Este se emplea para hacer una evaluación de la capacidad portante de las capas de afirmado mediante una correlación con el índice CBR. La correlación empleada para el cálculo del CBR a partir del PDC, es la desarrollada por la Universidad del Cauca [11].

\subsection{7. Índice de Condición Global o Serviciabilidad del Pavimento - OPI}

A partir de la información del índice de fallas superficiales (Modified Distress Rating - MDR) y del IRI, es posible calcular el valor del OPI (Overall Pavement Index) para cada una de las calzadas que conforman el segmento vial [12], [13].

\subsubsection{1. Índice de Fallas Superficiales - MDR}

El cálculo del MDR se basa en valores deducibles obtenidos de las respectivas curvas de fallas o pesos en función del grado de severidad y de la extensión del daño de acuerdo a la metodología PAVER [14]. Esta metodología fue desarrollada por el Cuerpo de Ingenieros del Ejército de los Estados Unidos. El valor deducible varía de 0 a 100 e indica el impacto que cada daño tiene sobre la condición del pavimento. Así, un valor de 0 en la curva, indica que el daño no tiene efecto en el comportamiento del pavimento, mientras un valor de 100 indica un daño de gran influencia. 
Los rangos asignados por la Secretaría de Obras públicas, para la calificación del MDR, son: MUY MALO (0-30), el pavimento se encuentra sumamente fallado y requiere una intervención de manera urgente y masiva sobre la calzada; MALO (30-50), presencia elevada de fallas de severidad baja a media, con una suma de la extensión total de las fallas inferior al $60 \%$ del área total mantenida; REGULAR (50-65), se requiere hacer un mantenimiento al pavimento, con el fin de evitar su rápida destrucción, la severidad de las fallas es baja y la suma de la extensión total de las fallas es inferior al $40 \%$ del área total de la calzada; BUENO (65-85), el pavimento requiere solamente una atención rutinaria, la severidad de las fallas es baja y la suma de la extensión total de las fallas es inferior al $25 \%$ del área total de la calzada; MUY BUENO (85100) el pavimento se encuentra en óptimas condiciones, sin presencia de fallas

El valor de MDR se calcula bajo la siguiente expresión basada en la Metodología Washington [12].

$$
M D R=100-\sqrt{\left(\sum p n_{i}{ }^{2}\right)}
$$

Donde "pn" es el peso de ponderación del daño según su severidad y extensión. Este cálculo, inicialmente, se basa en los valores deducibles obtenidos de las respectivas curvas o pesos en función del grado de severidad y de la extensión del daño de acuerdo con el Sistema PAVER.

Los valores deducibles se introducen en la fórmula antes mencionada, obteniéndose el valor del MDR. En el caso de vías con alto grado de deterioro, la raíz cuadrada de la sumatoria de los pesos al cuadrado puede resultar mayor que 100 , por lo cual al realizar el cálculo se obtendría valores negativos. Por definición, el MDR es un índice positivo, en estos casos, se asigna un valor $\mathrm{MDR}=0$.

Las tipologías de fallas analizadas en los pavimentos flexibles para el cálculo del MDR son: Abultamientos, Corrugaciones, Agrietamiento en bloque, Ahuellamiento, Baches, Descascaramiento, Exudación del asfalto, Grietas longitudinales y transversales, Hundimientos, Grietas en media luna, Peladuras, Piel de cocodrilo, Desintegración de bordes, Fisuramiento por reflexión, Parcheo, Brechas de servicios públicos.

El índice de fallas superficiales - MDR conjuntamente con la medida de regularidad IRI, conforman el índice de condición global del pavimento - OPI. La importancia del levantamiento de fallas, radica en que a partir del resultado de éstas se pueden programar intervenciones rutinarias o periódicas, puntuales 0 masivas, para conservar un nivel de servicio adecuado en cada una de las calzadas vehiculares. El deterioro de un pavimento se entiende como la serie de daños y manifestaciones superficiales de la capa de rodadura que perjudican la condición de circulación segura y confortable.

\subsubsection{Cálculo del Índice de Condición Global del Pavimento - OPI}

La Guía AASHTO [19] establece que "La serviciabilidad es la habilidad específica de una sección de pavimento para servir al tráfico". Hay dos formas de definir la serviciabilidad. La primera es basada en la medida del Índice de Rugosidad, el cual considera solamente la rugosidad del pavimento; este índice se denomina Índice de Serviciabilidad Presente del Pavimento PSI (Pavement Serviciability Index) y se calcula a través de la siguiente expresión:

$$
P S I=5 \times e^{0.198-0.00026 * I R I}
$$

Dónde: PSI es el Índice de Serviciabilidad del Pavimento; (e) es la base de logaritmos neperianos y el IRI es el Índice de Rugosidad Internacional

La otra forma de evaluar la serviciabilidad es a través de la evaluación combinada de la rugosidad y el estado superficial del pavimento a través de los daños superficiales, expresión que la AASHTO denomina Índice de Condición Global del Pavimento y que ha sido evaluada y calibrada por la empresa de consultoría TNM Limitada a lo largo de varios años de investigación, encontrando el Índice de Condición Global del Pavimento. El OPI representa una interrelación del estado superficial (desempeño funcional y estructural) y la condición del pavimento medido en términos de rugosidad (desempeño funcional), siendo, por lo tanto, un parámetro representativo del estado global del pavimento. La expresión encontrada para el OPI es:

$$
O P I=M D R \times(P S I / 5)^{0.22}
$$

Dónde: OPI es el Índice de Condición Global del Pavimento; MDR es el Índice de Fallas Superficiales; PSI es el Índice de Serviciabilidad del Pavimento

\subsubsection{Cálculo del número estructural $-\mathrm{SN}$}

El procedimiento referenciado de retro cálculo para la estimación del número estructural actual o efectivo en pavimentos flexibles, así como del módulo resiliente de la subrasante - Mr por la metodología AASHTO se detalla a continuación:

Se calcula el número estructural efectivo mediante la relación de la AASHTO [15]:

$$
S N_{\text {Efectivo }}=0.0045 * D * \sqrt[3]{E_{P}}
$$

Dónde: $\mathrm{SN}_{\text {efectivo }}$ es el número estructural efectivo existente en pulgadas; (D) Espesor total de las capas del pavimento sobre la subrasante, en pulgadas; (Ep) es el Módulo equivalente de la estructura del pavimento, en PSI (libras por pulgada cuadrada), determinado a través de la siguiente ecuación [8]. 


$$
d_{0}=1.5 * p^{*} a *\left\{\frac{1}{M_{r} \sqrt{1+\left(\frac{D}{a} \sqrt[3]{\frac{E_{p}}{M_{r}}}\right)^{2}}}+\left[\frac{1-\frac{1}{\sqrt{1+\left(\frac{D}{a}\right)^{2}}}}{E_{p}}\right]\right\}
$$

Dónde: (do) es la Deflexión central obtenida del inventario deflectométrico FWD); ( $p$ ) es la Presión aplicada en la medición de deflexiones, en PSI; (a) es el Radio del plato de carga del deflectómetro, en pulgadas; (D) es el Espesor total de las capas del pavimento sobre la subrasante, en pulgadas; (Ep)es el Módulo equivalente de la estructura del pavimento, en PSI y (Mr) es el Módulo resiliente de la subrasante, en psi, determinado por la relación[15]:

$$
M r=\frac{0.24 * P}{d_{r} * r}
$$

Dónde: $(P)$ es la Carga aplicada por el deflectómetro de impacto en libras; (dr) es la Deflexión Df6 medida a una distancia de $1.8 \mathrm{~m}$ del centro del plato de carga; $(r)$ es la Distancia desde el centro del plato de carga, en pulgadas.

El valor del CBR en pavimentos flexibles se calculó a partir del valor del $\mathrm{Mr}$ encontrado por retro cálculo, mediante la expresión. La importancia del cálculo de este parámetro $(\mathrm{Mr})$ es la correlación que existe con la capacidad portante de la subrasante que se determina mediante el CBR, evitando así procesos destructivos en el pavimento cuando se mide en campo mediante apiques [16]:

$\mathrm{Mr}=100 \times \mathrm{CBR}(\mathrm{kg} / \mathrm{cm} 2)$

\subsubsection{Parámetros Geométricos del Pavimento}

La información recopilada en campo corresponde a la ubicación y descripción general del segmento vial. La ubicación está referida a datos tales como nomenclatura, nombre de las vías generadoras de la nomenclatura y tipo de vía. Los datos de geometría tales como longitud, ancho, pendiente y número de carriles están referidos a las calzadas, bahías, bermas, andenes, cunetas, ciclorutas y barreras de protección. También se toman datos generales de señalización horizontal y vertical, cámaras, válvulas, sumideros, muros de contención y puentes. La geometría es importante para la definición de la sección transversal del segmento vial y la estimación de las cantidades de obra requeridas para las intervenciones.

\subsubsection{Tránsito Promedio Diario - TPD}

El Transito Promedio Diario - TPD se define como el número de autos, buses y camiones a partir del cual se determina la carga sobre el pavimento, mediante el número de ejes equivalentes de 8.2 toneladas. Mediante la determinación y asignación del TPD en cada segmento vial, es posible estimar la magnitud, composición y distribución de los flujos vehiculares en los diferentes corredores viales de la ciudad. Este enfoque permite determinar actividades de planeación estratégica tendientes a la optimización de los recursos destinados al mantenimiento vial.

Con el fin de determinar el TPD se caracterizan tres grupos de vehículos que son: TPD Autos, corresponde al valor de Tránsito Promedio de 24 horas de circulación de autos en la calzada inventariada. Los autos hacen referencia a todos los vehículos livianos.TPD Bus, corresponde al valor de Tránsito Promedio de 24 horas de circulación de buses en la calzada inventariada. Los buses incluyen los buses y busetas.TPD Camión, corresponde al valor de Tránsito Promedio de 24 horas de circulación de camiones en la calzada inventariada. Los camiones corresponden a todos los vehículos de carga con cuatro o más ruedas, de acuerdo con la siguiente clasificación de ejes: Camión C2: Camión de dos (2) ejes, Camión C3: Camión de tres (3) ejes, Camión C4: Camión de cuatro (4) ejes, Camión C5: Camión de cinco (5) ejes y Camión >C5: Camión de más de cinco (5) ejes.

La asignación del TPD se realizó mediante la zonificación de la ciudad, teniendo en cuenta las características relacionadas con especificaciones viales y los patrones de movilidad y físicos que las diferencian, tales como la topografía, rutas de buses, usos del suelo y usos del automóvil particular. La ciudad se caracterizó en seis (6) zonas homogéneas indicadas en la Figura 2.

La primera zona corresponde al corredor del rio (autopista urbana), vía de mayor importancia de la ciudad que atraviesa de sur a norte la ciudad, de topografía plana $y$, volúmenes altos de vehículos pesados.

La segunda zona es la Nor-Oriental, caracterizada por fuertes y pronunciadas pendientes en dirección de las calles, vías con bajas especificaciones, en cuanto a la movilidad predomina el transporte público formal e informal, la presencia de automóviles particulares no es significativa y con alta densidad poblacional de la zona.

La tercera zona es la Centro-Oriental, en donde se ubica el centro de la ciudad, con tránsito alto de vehículos de transporte público, se caracteriza por grandes avenidas de varias calzadas y por ser de topografía plana en el centro.

La cuarta zona es la Sur-Oriental, con altas y pronunciadas pendientes, con densidad baja de vías pero de alto nivel jerárquico, buenas especificaciones, volúmenes de transito altos en vehículos particulares, zona especialmente residencial y comercial.

La quinta zona es la Sur - Occidental, con pendientes moderadas y planas en su extensión, residencial, con algunos sectores industriales y comerciales, con avenidas importantes, de varias calzadas, alta movilidad y gran presencia de vehículos particulares y de transporte público. 
Finalmente, la sexta zona es la Nor-Occidental, con iguales características que la zona 2, es decir con altas pendientes, con presencia poco significativa de vehículos particulares, densidades altas de población y vías con bajas especificaciones.

Fig. 2.

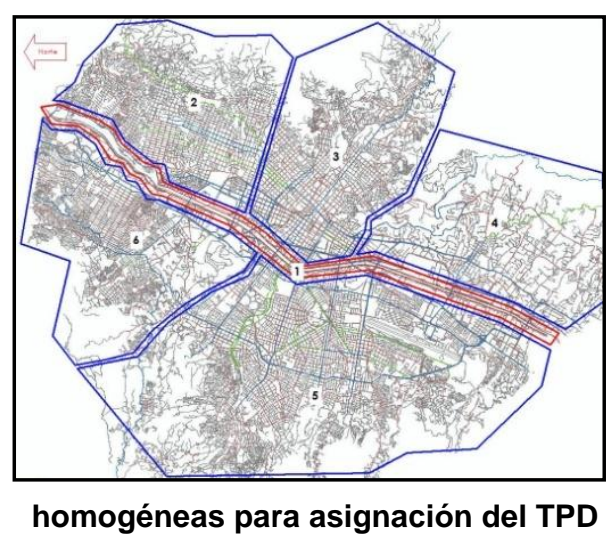

Zonas

Las vías de la ciudad se clasificaron de acuerdo a una serie de familias de vías típicas, que presentan condiciones muy similares en su nivel de demanda. Su identificación está en función de tres variables básicas: el componente físico, el componente funcional y el componente operativo. El componente físico se refiere a la geometría transversal del segmento vial de acuerdo al número de calzadas y carriles presentes en cada segmento y se clasifican con base en su capacidad. El componente funcional tiene en cuenta la jerarquía de la vía establecida en el Plan de Ordenamiento Territorial - POT, clasificando el segmento vial con base en la importancia de su ubicación dentro de la funcionalidad de la malla vial. El componente operativo refleja las diferencias que se presentan en los segmentos viales respecto de la composición vehicular esperada, con base en el uso del suelo y el estrato socio económico donde se encuentra el segmento.

La superposición de estos tres componentes permite clasificar familias de vías que cumplen con características comunes, especialmente con su localización en cada una de las zonas establecidas, lo cual posibilita el diseño de la toma de información de campo.

El número de calzadas resultante de las familias de vías según la jerarquía vial y el TPD se indica en la Tabla 1.

Tabla 1. Número de calzadas según TPD y jerarquía

\begin{tabular}{|l|c|c|c|c|}
\hline & \multicolumn{4}{|c|}{ Tránsito Promedio Diario TPD (anual) } \\
\hline $\begin{array}{c}\text { Jerarquía } \\
\text { Vial }\end{array}$ & $\begin{array}{c}<4.000 .00 \\
0\end{array}$ & $\begin{array}{c}4.000 .000- \\
8.000 .000\end{array}$ & 8.000 .000 & $\begin{array}{c}\text { Total } \\
\text { genera } \\
\text { I }\end{array}$ \\
\hline Autopista & 85 & 22 & 70 & 177 \\
\hline $\begin{array}{l}\text { Artería } \\
\text { Principal }\end{array}$ & 641 & 1540 & 728 & 2909 \\
\hline $\begin{array}{l}\text { Artería } \\
\text { menor }\end{array}$ & 404 & 161 & 42 & 607 \\
\hline Colectora & 4419 & 335 & 43 & 4797 \\
\hline Servicio & 12909 & 187 & 8 & 13104 \\
\hline
\end{tabular}

\begin{tabular}{|l|c|c|c|c|}
\hline & \multicolumn{3}{|c|}{ Tránsito Promedio Diario TPD (anual) } \\
\hline $\begin{array}{c}\text { Jerarquía } \\
\text { Vial }\end{array}$ & $\begin{array}{c}<4.000 .00 \\
0\end{array}$ & $\begin{array}{c}4.000 .000- \\
8.000 .000\end{array}$ & 8.000 .000 & $\begin{array}{c}\text { Total } \\
\text { genera } \\
\text { I }\end{array}$ \\
\hline Rural & 2495 & 2 & 0 & 2497 \\
\hline $\begin{array}{l}\text { Total } \\
\text { general }\end{array}$ & 20953 & 2247 & 891 & 24091 \\
\hline
\end{tabular}

\subsubsection{Cálculo del Número de ejes equivalentes $\mathbf{N}$}

Con los datos asignados de tránsito promedio diario, se determinó el número de ejes equivalentes de 8.2 ton en cada una de las calzadas. Los volúmenes de tránsito y su composición vehicular permiten el cálculo del Número de Ejes Equivalente, utilizando los factores de daño de cada tipo de vehículo y está dado por la siguiente expresión:

$$
\mathrm{N}=365^{*}\left(\mathrm{Va}{ }^{*} \mathrm{Fa}+\mathrm{Vb}{ }^{*} \mathrm{Fb}+\mathrm{Vc}{ }^{*} \mathrm{Fc}\right)
$$

Donde, Fa, Fb y Fc indican los factores de daño para automóviles, buses y camiones, respectivamente y $\mathrm{Va}$, $\mathrm{Vb}$ y Vc son volúmenes de vehículos que corresponden al valor del Tránsito Promedio Diario de autos, buses y camiones.

La determinación de los Factores Daño para este proyecto, incluidos en el cálculo del número de ejes equivalentes, se realizó a partir de los resultados del estudio de tránsito en cuanto a distribución del parque automotor y empleando los factores de equivalencia promedio utilizados más frecuentemente (Universidad del Cauca - 1996), los cuales se indican en la Tabla 2 [17].

Tabla 2. Factores de equivalencia por tipo de vehícu
\begin{tabular}{|c|c|}
\hline $\begin{array}{c}\text { Tipo de } \\
\text { Vehículo }\end{array}$ & $\begin{array}{c}\text { Factor de equivalencia } \\
\text { (Universidad del Cauca - } \\
\text { 1996) }\end{array}$ \\
\hline Buseta & 0.05 \\
\hline Bus & 1.00 \\
\hline C2 P & 1.14 \\
\hline C2G & 3.44 \\
\hline C3 - C4 & 6.73 \\
\hline C5 & 4.40 \\
\hline > C5 & 4.72 \\
\hline
\end{tabular}

En cada una de las estaciones de aforo, en las cuales se obtuvo un valor discriminado de tránsito para cada tipo de vehículo, se llevó a cabo el cálculo de los factores daño, multiplicando cada factor por el valor porcentual de tránsito por cada tipo de vehículo. Es decir, el factor daño de los buses, que incluye buses y busetas, y el de camiones se calcularon mediante las fórmulas:

$$
\mathrm{Fb}=(\% \text { TPDbusetas * } 0.05)+(\% \text { TPDbuses * } 1.00)
$$

$\mathrm{FC}=(\%$ TPDC2P * 1.14$)+(\% \mathrm{TPDC} 2 \mathrm{G} * 3.44)+(\%$ TPDC3-C4 * 6.73) $+(\%$ TPDC5 * 4.40$)+(\%$ TPD $>$ C5 * 4.72) 
Donde C2P, C2G, C3-C4-C5, es la caracterización de los camiones de acuerdo al tipo de ejes, ya sea sencillo, doble o tándem [18].

Para los autos, no se tienen factores de equivalencia, sin embargo, para no excluir el mínimo valor de aplicaciones que este puede aportar sobre la estructura de pavimento, se asumirá un valor mínimo de 0.001 . Después de tener calculados todos los factores de daño, se procedió a calcular el promedio de todos los movimientos evaluados en campo. El resultado obtenido es el siguiente: $\mathrm{Fa}=0.001, \mathrm{Fb}=0.47$ y $\mathrm{Fc}=$ 2.52; lo cual indica claramente que los camiones afectan en una escala ponderada de 2.0 veces más que un bus, ya que las cargas concentradas de sus ejes influyen notablemente en el daño y la aparición de las fallos superficiales. Por ello en los diseños de pavimentos flexibles se considera como parámetro de diseño el número equivalente $(\mathrm{N})$ con una carga de 8.2 ton durante su vida útil.

\subsection{MATRIZ DE DECISIONES}

El segundo elemento a considerar en la implementación del Sistema de Gestión Vial, es la elaboración de una Matriz de Decisiones, cuyo objetivo básico consiste en establecer la intervención requerida en un segmento vial para mantener un nivel de servicio adecuado y proteger el patrimonio vial en un cierto período de tiempo. Con los datos de diagnóstico, se hace la gestión sobre cada uno de los elementos, definiendo previamente unos criterios apropiados del estado de los elementos.

\subsubsection{Filosofía de la Matriz de decisiones}

Las estructuras de pavimento son construidas con el objetivo de servir al tránsito en forma segura, confortable y eficiente, sin embargo, el costo en que deben incurrir las agencias viales para su construcción es demasiado alto y el retorno de dicha inversión solo es justificable en la medida que el volumen de bienes y servicios transportados justifiquen dicha inversión, la cual se cuantifica en el concepto denominado Patrimonio Vial. Este concepto corresponde a la suma del costo de tres elementos básicos: Valor del terreno, que hace referencia al valor del espacio o franja que corresponde al derecho de vía en la cual se construye la calzada; el valor del pavimento, que corresponde al valor actual de la superficie de rodadura, teniendo en cuenta el deterioro que presenta dicha superficie por acción de las cargas vehiculares, efectos ambientales como el clima y la deficiencia en las especificaciones técnicas de los materiales o procesos constructivos; y el valor de los elementos o infraestructura asociada al pavimento, que corresponde al costo de los materiales, equipos, transporte y mano de obra necesarios para la construcción de los mismos, elementos indispensables para la operación de la estructura de pavimento en términos estructurales, funcionales y de seguridad, costo depreciado de acuerdo a su condición estructural y funcional.

De otra parte, se encuentra que tanto el pavimento como los elementos asociados presentan desde el punto de vista funcional y estructural una evolución o deterioro a través del tiempo generado por la acción de las cargas, el clima y otros factores, lo cual implica que con la sola construcción de la infraestructura no es posible servir de manera eficiente a los usuarios durante todo el período de vida útil estimado en el diseño. Es decir, se hace necesario realizar acciones de mantenimiento para conservar el valor del patrimonio vial en un estándar mínimo que no implique a posteriori inversiones mayores para recuperar el patrimonio perdido por ausencia de acciones preventivas.

Bajo los conceptos anteriormente descritos nace un nuevo concepto denominado "Gestión de Pavimentos" el cual se puede definir como el conjunto de operaciones que tienen como objetivo conservar por un período de tiempo las condiciones de seguridad, comodidad y capacidad estructural adecuadas para la circulación, soportando las condiciones climáticas y de entorno en que su ubica la malla vial estudiada. Todo lo anterior, con el objetivo de minimizar el costo monetario, social y ecológico que implica reconstruir una malla que ha llegado a su valor mínimo.

Los recursos de que dispone la Administración de Medellín para implementar un modelo de mantenimiento adecuado que cubra la totalidad del área pavimentada son insuficientes, al menos mientras se modifica el paradigma de ubicar mayores recursos para el mantenimiento de los pavimentos. Esta situación conduce a que el modelo o Matriz de Decisiones a estructurar, al menos inicialmente, debe ser flexible y sostenible a través del tiempo y que además permita ser retroalimentado con el objetivo de establecer posibles mejoras de acuerdo a la experiencia del comportamiento de las intervenciones directamente en campo. De igual forma, debe permitir en el corto y mediano plazo, la inversión de los pocos recursos disponibles en las zonas con mejor estado, cubriendo la mayor área de pavimento posible, elevando progresivamente su nivel de servicio. Lo anterior, bajo parámetros de medición y metodologías de auscultación e intervención con base en tecnología de punta, para que posteriormente, a largo plazo, los estándares de estado superficial y estructural se eleven y se puedan cubrir mayores áreas.

Por lo anterior, el concepto de "rehabilitación" o "reconstrucción" concebido en la Matriz de Decisiones se limita a condiciones superficiales y estructurales extremas, dado que mientras que las mismas se presentan se considera viable potencializar la respuesta de los pavimentos existentes mediante acciones anuales de mantenimiento rutinario y periódico.

Se considera que las acciones de mantenimiento a implementar deben ser anuales dado que los pavimentos son estructuras dinámicas y por lo tanto requieren atención periódica. No obstante lo anterior, si un pavimento se encuentra en malas condiciones y es intervenido de forma masiva, ya sea mediante una 
Ing. USBMed, Vol. 3, No. 2, Julio-Diciembre 2012

acción preventiva o correctiva, se entiende que a corto y mediano plazo en los años posteriores solo requerirá de intervenciones de carácter rutinario hasta que alcance un nivel de servicio tal que requiera una intervención mayor.

Por lo tanto, el objetivo básico de la Matriz de Decisiones consiste en establecer la intervención requerida en un segmento vial para mantener un nivel de servicio adecuado y proteger el Patrimonio Vial en un cierto período de tiempo, a partir de la combinación de diferentes variables para cada tipo de pavimento (flexible, rígido y articulado). Dentro de estas variables se encuentran la jerarquía del segmento de acuerdo a los criterios establecidos por el Plan de Ordenamiento Territorial - POT, el nivel de tráfico proyectado que solicita el segmento, su condición estructural y su condición funcional.

\subsubsection{Rangos de la Matriz de Decisiones}

Los rangos establecidos en la Matriz de Decisiones combinan los parámetros técnicos TPD (Tabla 3), el SN (Tabla 4) y el OPI (Tabla 5). Estos rangos fueron definidos y ajustados de acuerdo con los estándares de la Secretaría de Infraestructura Física.

La clasificación por TPD (Tránsito Promedio Diario) proyectado a un (1) año para la Matriz de Decisiones se muestra en la Tabla 2.

Tabla 3. Clasificación del TPD

\begin{tabular}{|c|c|}
\hline Clasificación & $\begin{array}{c}\text { Rangos del Tránsito Promedio } \\
\text { DiarioTPD(unidades) }\end{array}$ \\
\hline Bajo & TPD $<4.000 .000$ \\
\hline Medio & TPD $(4.000 .000-8.000 .000)$ \\
\hline Alto & TPD $>8.000 .000$ \\
\hline
\end{tabular}

La clasificación del número estructural SN, el cual depende para estructuras de pavimento con espesor constante y deflexiones centrales, tiene los rangos indicados en la Tabla 3.

Tabla 4. Clasificación del SN

\begin{tabular}{|c|c|}
\hline Clasificación & $\begin{array}{c}\text { Rangos del número estructural SN } \\
\text { (pulgada) }\end{array}$ \\
\hline Bajo & $\mathrm{SN}<3$ \\
\hline Medio & $\mathrm{SN}(3-4)$ \\
\hline Alto & $\mathrm{SN}>4$ \\
\hline
\end{tabular}

La clasificación de las vías de acuerdo a la serviciabilidad es a través de la evaluación combinada de la rugosidad (IRI) y el estado superficial del pavimento (MDR) a través de los daños superficiales, expresión que la AASHTO denomina Índice de Serviciabilidad Global (OPI).

Los rangos de OPI considerados para la Matriz de Decisiones se indican en la Tabla 4.
Tabla 5. Clasificación del OPI

\begin{tabular}{|c|c|}
\hline Clasificación & $\begin{array}{c}\text { Índice de Serviciabilidad Global } \\
\text { OPI }\end{array}$ \\
\hline Muy Bueno & $85-100$ \\
\hline Bueno & $65-84$ \\
\hline Regular & $40-64$ \\
\hline Malo & $0-39$ \\
\hline
\end{tabular}

Una vez combinadas las variables se procede a la definición de las intervenciones requeridas. Para ello se contempla la patología de daños superficiales presentados, las cuales difieren dependiendo de la causa que la produce. También se considera cuando el pavimento no tiene fallas superficiales, para cuyo caso no requiere intervención.

Para los pavimentos flexibles se han establecido cuatro (4) generadores de causas para las patologías superficiales como son: problemas estructurales, problemas geotécnicos y de confinamiento, problemas de la mezcla asfáltica y reparaciones de la superficie.

Una vez clasificado el segmento vial de acuerdo con las variables analizadas, y establecido el daño o los daños presentes, se definen las intervenciones requeridas para mantener el nivel de servicio de la vía en un nivel óptimo durante un cierto período de tiempo.

\subsubsection{Intervenciones generadas por la Matriz de Decisiones}

Las intervenciones requeridas para mantener el nivel de servicio de la vía se clasifican en cuatro (4) grandes grupos y se identifican con un determinado color en la Matriz. El Mantenimiento Rutinario, se identifica con el color verde, corresponde a las acciones preventivas sobre la calzada y que son necesarias para lograr que la curva de deterioro de cada uno de los parámetros se comporte de acuerdo al diseño del pavimento. El Mantenimiento Periódico Puntual, se identifica con el color amarillo, corresponde a las acciones preventivas sobre el pavimento con el objetivo de solucionar daños puntuales presentes en el pavimento. El Mantenimiento Periódico Masivo, se identifica con el color naranja, corresponde a las acciones preventivas sobre el $100 \%$ del pavimento con el objetivo de solucionar daños generalizados en la superficie del pavimento que abarcan áreas importantes del segmento vial. La Rehabilitación o Reconstrucción, se identifica con el color rojo, corresponde a acciones reactivas frente a deficiencias estructurales severas frente al nivel de solicitación de carga de la calzada vehicular, reparando el $100 \%$ del mismo.

En la Figura 3, se observa el proceso de deterioro progresivo de un pavimento en el tiempo, para el cual se tomó como referente el modelo de deterioro HDM-4 (Metodología del Banco Mundial), que fue calibrado para la ciudad de Medellín [19]. 


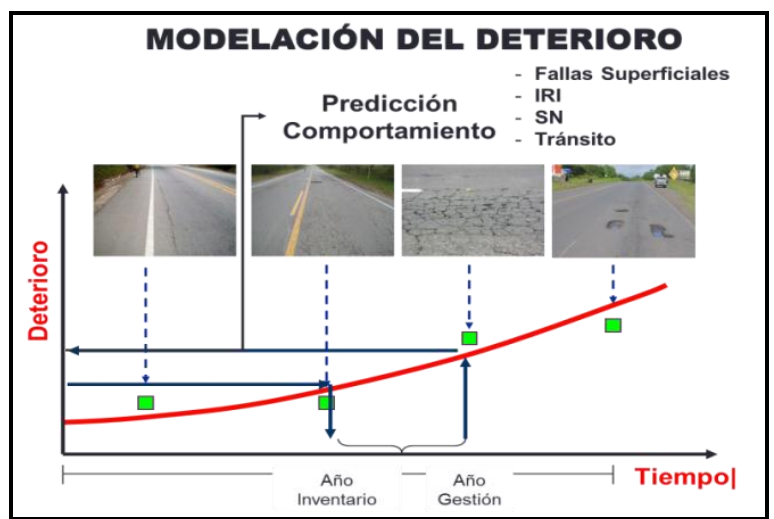

Fig. 3. Modelación del deterioro [5]

El alcance de las intervenciones futuras a partir de la implementación de las intervenciones generadas por la Matriz de Decisiones, se muestran en la Figura 4.

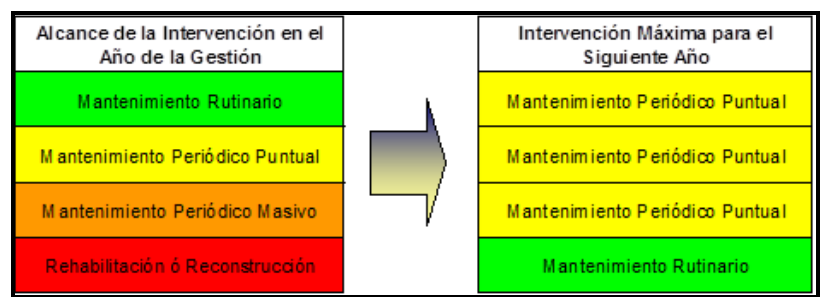

Fig. 4. Alcance de las intervenciones [5]

De igual forma, es importante anotar que esta Matriz se constituye en una herramienta de "gestión" la cual permite cuantificar de manera adecuada los recursos necesarios para la conservación del Patrimonio Vial a partir de los datos de diagnóstico levantados directamente en campo. Las intervenciones en Pavimento Flexible tienen relación con las especificaciones técnicas normalizadas, recomendadas para su correcta implementación a nivel nacional, y se indican en la Tabla 6.

Tabla 6. Intervenciones en pavimento flexible

\begin{tabular}{|c|c|}
\hline Descripción de la intervención & Tipo \\
\hline Mantenimiento rutinario de la calzada & \multirow{6}{*}{ Puntual } \\
\hline Sello de fisuras & \\
\hline Parcheo de huecos ó parcheo elaborado & \\
\hline Re nivelación de la carpeta asfáltica & \\
\hline $\begin{array}{lll}\begin{array}{l}\text { Confinamiento de la estructura y re } \\
\text { nivelación de carpeta }\end{array} & & \\
\end{array}$ & \\
\hline Corrección de daños y slurryseal & \\
\hline $\begin{array}{l}\text { Corrección de daños y micro aglomerado } \\
\text { en caliente }\end{array}$ & \multirow{4}{*}{ Masivo } \\
\hline Parcheo y sobre carpeta de $5 \mathrm{~cm}$ & \\
\hline $\begin{array}{l}\text { Fresado hasta espesor requerido, } \\
\text { reposición de la carpeta asfáltica y sobre } \\
\text { carpeta de } 8 \mathrm{~cm}\end{array}$ & \\
\hline Reposición total de la carpeta & \\
\hline $\begin{array}{l}\text { Estudios y diseños de rehabilitación de la } \\
\text { estructura de pavimento }\end{array}$ & Masivo \\
\hline
\end{tabular}

\section{METODOLOGÍA}

La implementación del Sistema de Gestión Vial está soportada en una Base de Datos Geográfica anidada en una Geodatabase corporativa del Municipio de Medellín, bajo una plataforma SIG y administrado bajo el software de ArcGIS.

\subsection{Base de Datos}

Un elemento fundamental para la estructuración de la Base de Datos es el Modelo Entidad - Relación, el cual se presenta en una forma resumida en la Fig.5.Las capas o reglas de negocio se llevan a cabo mediante el desarrollo de módulos Java 1.6. La base de datos es manejada por motor Oracle 11G.

\begin{tabular}{|l|l|}
\hline \multicolumn{1}{|l|}{} & Segmento \\
& Identificador del segmento \\
\hline & Longitud \\
& Nodos \\
& Comuna-Barrio \\
\hline Calzada & Fallas \\
\hline Identificador Calzada & Identificador \\
\hline Segmento & Calzada \\
\hline Ancho & Orden \\
\hline Superficie & Tipo \\
\hline Condiciones de servicio & \\
\hline Condiciones de transito & \\
\hline Condiciones estructurales & \\
\hline
\end{tabular}

Fig. 5. Resumen Modelo Entidad - Relación

\subsection{Implementación del SIG para la Visualización y Análisis}

Otro elemento constitutivo del sistema es la visualización gráfica de todas las aplicaciones, utilidades y desarrollos, en un ambiente SIG, herramienta de alto poder de análisis, interpretación y visualización, que emplea tecnología de avanzada mediante hardware y software especializados. EI SIG permite integrar datos alfanuméricos con la información geográfica, realizar análisis multicriterio, apoya el establecimiento de la toma de decisiones permitiendo la optimización de los recursos.

En la implementación del Sistema de Gestión Vial se tiene un modelo distribuido, con un servidor de aplicaciones; éste se comunica e interactúa con tres servidores adicionales, el primero es el servidor de base de datos en Oracle y el SDE, el segundo es el servidor de archivos donde reposan las fotos y videos; y el tercero es el servidor de ArcGIS Server. Todos los servidores se encuentran vinculados a través de la Web.

El esquema distribuido permite tener una mayor disponibilidad de la información, ya que cada servidor contribuye a sustentar los recursos que requiera la Gestión, ya sea de almacenamiento y procesamiento de información o de aplicaciones. Adicionalmente el esquema distribuido brinda mayor agilidad en la consulta de información al momento de realizar peticiones a la interfaz; permitiendo una infraestructura modular, versátil y con interoperabilidad. Con la evolución de las nuevas aplicaciones y el esquema distribuido, se logra mayor integración de los servidores y un uso más productivo de la información.

El esquema distribuido brinda escalabilidad $y$ crecimiento, es decir las redes se pueden ampliar, 
aumentando el número de módulos o sustituyendo por otros más potentes, es fácilmente adaptable a nuevos problemas y soluciones del hardware.

El esquema empleado para el desarrollo o arquitectura del sistema, está a nivel de bases de datos, de bienes y servicios y en las aplicaciones. La capa de presentación del sistema de Gestión se desarrolló en Flex A.P.I. versión 4.5.1, como se ilustra en la Figura 6.

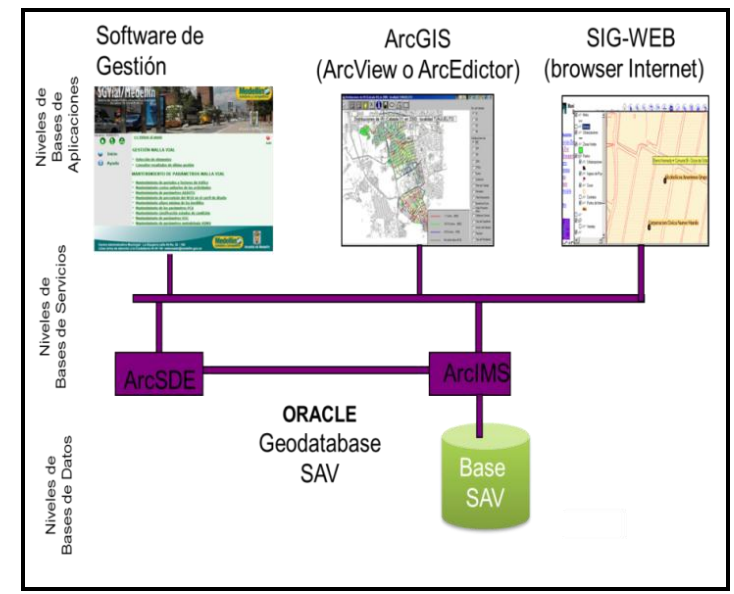

Fig.6. Arquitectura del Sistema de Gestión Vial

A nivel de bases de datos el repositorio de toda la información recopilada del inventario se encuentra en una Geotadabase corporativa para la Secretaría de Infraestructura Física, en la cual se encuentra la cobertura vial (Capa Segmento), que es el elemento geográfico del sistema.

La plataforma geográfica definida como estándar para la Secretaría es ESRI, en todos sus productos (ArcIMS, ArcSDE, ArcView, Arclnfo, ArcEditor). A nivel de bases de servicios el repositorio de almacenamiento de objetos Geográficos es ArcSDE para la parte de servidores e interfaz con los centros de servicios de ArcGIS. El publicador Intranet o Internet a nivel georreferenciado y de publicación de mapas es ArclMS. A nivel de las aplicaciones como herramientas de análisis técnico multicriterio, explotación y minería de datos se tiene los desarrollos de ArcGIS (ArcView, ArcEditor, Arclnfo) versión 10.1, el software de gestión en Java y los browser web. Finalmente, se cuenta con una plataforma e infraestructura tecnológica a nivel de hardware que asegura el funcionamiento del Sistema de Información Geográfica del aplicativo [20].

\section{RESULTADOS}

\subsection{Análisis de los datos de inventario}

La mayor importancia del proyecto de "Inventario y Diagnóstico de la Malla Vial de la ciudad de Medellín" radicó en el diagnóstico de la malla vial y la respectiva asignación de intervenciones en cada segmento, de acuerdo con la Matriz de Decisiones propuesta; se presenta un análisis estadístico de la información en las calzadas vehiculares de la ciudad.

En total, se recolectó información de 22.646 segmentos viales, en los cuales se encontraron 24.091 calzadas vehiculares, esto es debido a que hay segmentos viales que tienen más de una calzada por tener una mayor jerarquía vial, como por ejemplo las autopistas urbanas y arterias principales. La longitud total levantada en información fue de $2.053 \mathrm{Km}$-calzada. La distribución de las calzadas y su respectiva longitud, de acuerdo al tipo de pavimento y a la jerarquía vial, se presenta en la Tabla 7. En el presente inventario, sólo se tuvieron en cuenta las calzadas vehiculares.

Tabla 7. Número y Longitud de calzadas según el tipo de pavimento

\begin{tabular}{|l|c|c|}
\hline $\begin{array}{c}\text { Tipo de } \\
\text { Pavimento }\end{array}$ & $\begin{array}{c}\text { Número de } \\
\text { Calzadas }\end{array}$ & $\begin{array}{c}\text { Longitud de } \\
\text { Calzadas } \mathbf{( K m -} \\
\text { calzada) }\end{array}$ \\
\hline Flexible & 21.327 & $1.789,9$ \\
\hline Rígido & 1.788 & 125.4 \\
\hline Articulado & 115 & 8,6 \\
\hline Afirmado & 290 & 40,8 \\
\hline Otros & 571 & 124.3 \\
\hline TOTAL & 24.091 & 2.053 \\
\hline
\end{tabular}

Como se observa en la Figura 7, en el inventario de la malla vial se identificaron cinco tipos de pavimento o superficie de rodadura: flexible, rígido, articulado, afirmado y otros. El pavimento flexible representa el $89 \%$ del total, razón por la cual la mayoría de intervenciones están enfocadas hacia ese tipo de superficie. En segundo lugar está el pavimento rígido con $7 \%$ y el pavimento articulado representa sólo el $1 \%$.

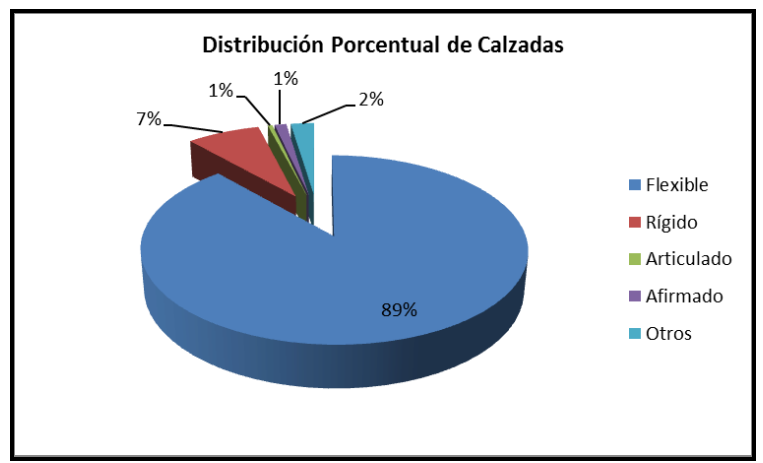

Fig. 7. Distribución porcentual de calzadas de acuerdo al tipo de pavimento

Similarmente, en la Tabla 8, se presenta la discriminación realizada para las calzadas vehiculares y los segmentos viales de acuerdo con el tipo de jerarquía vial entregado en la cobertura. Las vías identificadas como autopista, corresponden a las calzadas adyacentes al corredor del río en ambos costados.

Tabla 8.Número de calzadas vehiculares y segmentos según el tipo de vía

\begin{tabular}{|c|c|c|}
\hline Tipo de Vía & $\begin{array}{c}\text { Número de } \\
\text { Calzadas }\end{array}$ & $\begin{array}{c}\text { Número de } \\
\text { Segmentos }\end{array}$ \\
\hline Autopista & 177 & 172 \\
\hline Arteria Principal & 2.909 & 1.962 \\
\hline Arteria Menor & 607 & 472 \\
\hline Colectoras & 4.797 & 4.558 \\
\hline Servicio & 13.104 & 12.987 \\
\hline Rurales & 2.497 & 2.495 \\
\hline TOTAL & 24.091 & 22.646 \\
\hline
\end{tabular}


De igual manera, para la misma clasificación por tipo de vía, se determinó la longitud de las calzadas correspondiente a cada tipo como se indica en la Tabla 9.

Tabla 9. Longitud de calzadas según el tipo de vía

\begin{tabular}{|c|c|}
\hline Tipo de Vía & $\begin{array}{c}\text { Longitud de Calzadas } \\
\text { (Km-calzada) }\end{array}$ \\
\hline Autopista & 26,6 \\
\hline Arteria Principal & 296,5 \\
\hline Arteria Menor & 54,5 \\
\hline Colectoras & 394,1 \\
\hline Servicio & 963,1 \\
\hline Rurales & 318,4 \\
\hline TOTAL & 2.053 \\
\hline
\end{tabular}

En la Figura 8 se observa la distribución porcentual de la longitud de calzadas según el tipo de vía. Es así como el $47 \%$ corresponde a las vías de servicio, en segundo lugar están las vías colectoras con el 19\%, seguidas de las vías rurales con el 16\%, las arterias principales con el 14\%, las arterias menores con el $3 \%$ y finalmente las autopistas con el 1\%, siendo éstas últimas las vías de mayor jerarquía de la ciudad.

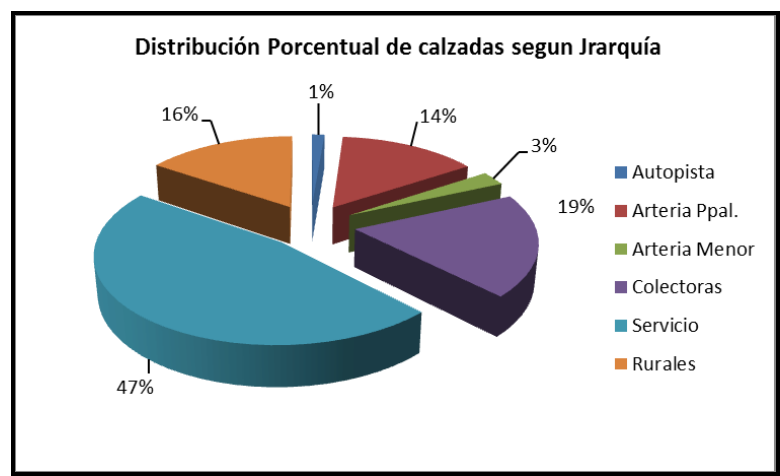

Fig. 8. Distribución porcentual de calzadas de acuerdo al tipo de vía POT

El mayor porcentaje de segmentos, correspondió a vías colectoras y de servicio. Esto no implica que la mayor longitud de inventario corresponda a este tipo de jerarquías, ya que es claro que las calzadas de mayor longitud estuvieron en la autopista. Las vías colectoras y las vías de servicio, en un alto porcentaje son segmentos de una sola calzada y esto se puede apreciar en el resumen que se presenta en la Fig. 9, donde se aprecia la configuración de la sección transversal del total de segmentos evaluados. Igual sucede con las vías rurales.

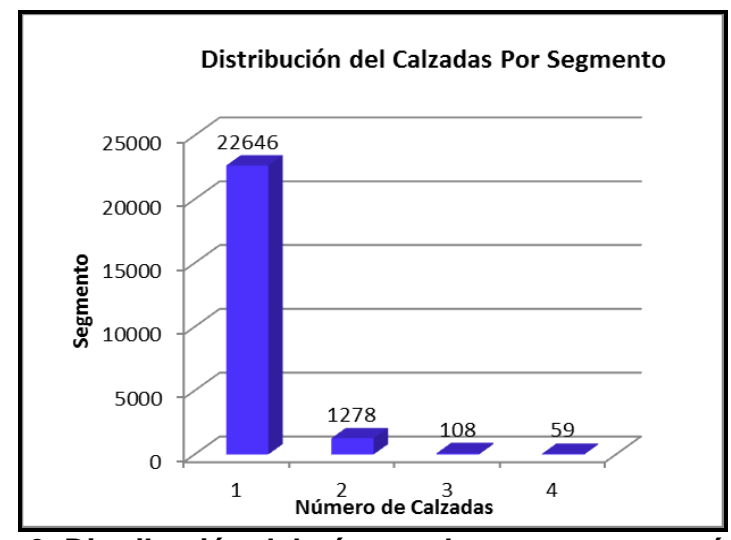

Fig. 9. Distribución del número de segmentos según la configuración de la sección transversal

\subsection{Resultado de la corrida de la matriz}

Para la corrida de la matriz se utilizaron las calzadas vehiculares con superficie en pavimento flexible por cada tipo de jerarquía vial, para obtener finalmente el tipo de intervención.

La longitud de calzadas y número de segmentos en pavimento flexible por tipo de jerarquía vial, se indica en la Tabla 10. De ésta longitud, el $49 \%$ corresponde a vías de servicio, el $22 \%$ a vías colectoras, luego el $15 \%$ a vías arterias principales, el 10\% a vías rurales, el 3\% a vías arterias menores y finalmente el $1 \%$ a las autopistas.

Tabla 10. Número de segmentos y longitud de calzadas según el tipo de vía

\begin{tabular}{|c|c|c|}
\hline Tipo de Vía & $\begin{array}{c}\text { Número de } \\
\text { Segmentos }\end{array}$ & $\begin{array}{c}\text { Longitud de } \\
\text { Calzadas (Km- } \\
\text { calzada) }\end{array}$ \\
\hline Autopista & 177 & 26.6 \\
\hline Arteria Principal & 2606 & 268.1 \\
\hline Arteria Menor & 606 & 54.3 \\
\hline Colectora & 4720 & 388.1 \\
\hline Servicio & 11708 & 875.3 \\
\hline Rural & 1510 & 177.5 \\
\hline TOTAL & 21.327 & $1.789,9$ \\
\hline
\end{tabular}

Dentro del grupo de calzadas en pavimento flexible, la matriz arrojó la clasificación del estado de las vías según el rango de $\mathrm{OPI}$, como se indica en la Tabla 11. Se encontró que el $41 \%$ está en regular estado, el $30 \%$ en mal estado, el $21 \%$ en buen estado y el $7 \%$ es excelente; sin embargo, sumando los estados bueno y excelente, se contabiliza el $28 \%$ de las vías en esas condiciones.

Tabla 11. Longitud y porcentaje de intervenciones generadas según los rangos de OPI

\begin{tabular}{|c|r|r|c|}
\hline OPI & $\begin{array}{c}\text { Longitud de } \\
\text { Calzadas (Km- } \\
\text { calzada) }\end{array}$ & Porcentaje & Estado \\
\hline $0-39.9$ & 550.9 & $30.8 \%$ & Malo \\
\hline $40-64.9$ & 740.8 & $41.4 \%$ & Regular \\
\hline $65-84.9$ & 368.9 & $20.6 \%$ & Bueno \\
\hline $85-100$ & 129.3 & $7.2 \%$ & Excelente \\
\hline TOTAL & 1789.9 & $100.0 \%$ & \\
\hline
\end{tabular}


Análogamente, la matriz arrojó la clasificación del estado de las vías en pavimento flexible según el rango de IRI, como se indica en la Tabla 12. Como se puede observar, este criterio duplica el porcentaje de estado excelente con el $15 \%$ y disminuye el porcentaje de estado bueno con el $7 \%$ con relación al criterio OPI, pero ambos sumados contabilizan el $22 \%$, que es similar al arrojado por este criterio. Luego viene el estado regular con el 53\%, ampliando el margen de aceptabilidad en comparación con el OPI y finalmente el estado malo con el $26 \%$.

Tabla 12. Longitud y porcentaje de intervenciones generadas según los rangos de IRI

\begin{tabular}{|l|r|r|l|}
\hline \multicolumn{1}{|c|}{ IRI } & $\begin{array}{c}\text { Longitud de } \\
\text { Calzadas (Km- } \\
\text { calzada) }\end{array}$ & Porcentaje & Estado \\
\hline 12 a 15 & 391.8 & $26.3 \%$ & Malo \\
\hline 5.5 a 11.99 & 946.2 & $52.5 \%$ & Regular \\
\hline 4.81 a 5.49 & 130.8 & $6.5 \%$ & Bueno \\
\hline 0 a 4.8 & 321.1 & $14.6 \%$ & Excelente \\
\hline TOTAL & 1789.9 & $100 \%$ & \\
\hline
\end{tabular}

Los resultados discriminados de acuerdo al tipo de intervención, arrojados por la matriz de decisiones se observan en el Anexo No. 1

\section{CONCLUSIONES}

Mediante la aplicación de los Sistemas de Información Geográfica - SIG al proceso de Gestión de la Malla Vial de la ciudad de Medellín, se caracterizaron y analizaron los elementos más importantes de su infraestructura y se identificaron los parámetros técnicos y geométricos que la conforman.

Estos elementos y parámetros se integraron espacialmente sobre una Geodatabase corporativa, que permite el despliegue de diferentes capas temáticas de estudio, tales como la red vial, topografía, usos del suelo, red hídrica, catastro, equipamientos colectivos, espacios públicos, división administrativa, sistemas de transporte, obras de infraestructura, tipos de superficie de rodadura, estados de condición, jerarquía vial, tipo de tráfico, pendientes, tipos de intervención, entre otros. En general, un conjunto de información que permite al usuario de los SIG disponer de un mejor entorno visual, que le facilita el análisis de la información.

De esta manera y teniendo como base la Matriz de Decisiones, que se desarrolló atendiendo los estándares de la malla vial de la ciudad, se realizaron procesos complejos de análisis multicriterio y se generaron diferentes recomendaciones de intervención. Las intervenciones generadas por la Matriz de Decisiones se obtuvieron luego de los procesos de análisis del modelo de deterioro HDM-4, el cual fue calibrado para la ciudad, teniendo en cuenta los criterios de priorización establecidos.

Entre los resultados obtenidos, se puede destacar que el $89 \%$ de las calzadas de la ciudad corresponden a pavimentos flexibles, razón por la cual la mayoría de intervenciones están enfocadas hacia ese tipo de superficie. En segundo lugar está el pavimento rígido con $7 \%$ y el pavimento articulado representa sólo el $1 \%$.

Del total de los $2.053 \mathrm{Km}$-calzada de longitud de la malla vial, según el tipo de vía o la jerarquía vial, el $47 \%$ corresponde a las vías de servicio, en segundo lugar están las vías colectoras con el 19\%, seguidas de las vías rurales con el $16 \%$, las arterias principales con el $14 \%$, las arterias menores con el $3 \%$ y finalmente las autopistas con el 1\%, siendo éstas últimas las vías de mayor jerarquía de la ciudad.

La Matriz de Decisiones arrojó que la clasificación del estado según el rango de IRI para las vías en pavimento flexible, es de $15 \%$ en estado excelente, $7 \%$ en estado bueno, $53 \%$ en estado regular y $26 \%$ en estado malo.

Finalmente, con los resultados obtenidos, se analizaron los datos estadísticos del inventario de la infraestructura y los mapas de las intervenciones requeridas en la gestión de la malla vial. Estos resultados, dinámicos en el tiempo, constituyen la herramienta principal de gestión de la Secretaría de Infraestructura Física. Ellos son la base para tomar decisiones y optimizar los recursos en la solución de un problema en particular y en la planificación del mantenimiento de la malla vial de la ciudad, lo que permite ofrecer un mejor servicio a la comunidad.

\section{REFERENCIAS}

[1]P. Álvarez, F. López-Rodríguez, J. L. Canito, F. J. Moral \& A. Camacho. "Development of a measure model for optimal planning of maintenance and improvement of roads".Computers \& Industrial Engineering, Vol. 52, Issue 3, pp. 327-335, April 2007.

[2]J. Coutinho-Rodrigues, A. Simão \& C. H. Antunes. "A GISbased multicriteria spatial decision support system for planning urban infrastructures". Decision Support Systems, Vol. 51, Issue 3, pp.720-726, June 2011.

[3]W. Chen, J. Yuan \& M. Li. "Application of GIS/GPS in Shanghai Airport Pavement Management System".Key Laboratory of Road and Traffic Engineering of the Ministry of Education, Tongji University, Shanghai 200092, P.R. China, Shanghai Airport Authority, Shanghai 200335, P.R. China.

[4]X. Wang. "Integrating GIS, simulation models, and visualization in traffic impact analysis". Computers, Environment and Urban Systems, Vol. 29, Issue 4, pp. 471-496, July 2005.

[5]TNM TECHNOLOGY \& MANAGEMENT. Online [Nov. 2011].

[6]W. A. Robertson, N. Sauceda \& R. Olive. "Condicion de las calles, evaluación de la infraestructura vial". Departamento de Obras Públicas, Agencia de Mantenimiento de Calles. Los Angeles, Septiembre de 2008. 
[7]M. W. Sayers \& S. M. Karamihas. "The Little Book of Profiling. Basic Information about Measuring and Interpreting Road Profiles". The Regent of the University of Michigan. Online [September. 1998].

[8]U.S. Department of Transportation Federal Highway Administration. "Geotechnical Aspects of Pavements Reference Manual”, Online [Nov. 2012].

[9]U.S. Department of Transportation Federal Highway Administration. "Ground Penetrating Radar for Measuring Pavement Layer Thickness", Publication Number: FHWA-HIF-00-015. 2000. Online [Nov. 2012].

[10] Mastrad, Quality and Test Systems. Online [Nov. 2012].

[11] Instituto Nacional de Vías, Universidad de Cauca, "Método De Ensayo Normal Para El Uso Del Penetrómetro Dinámico De Cono En Aplicaciones De Pavimentos A Poca Profundidad". Online [Nov. 2012].

[12] U.S. Department of Transportation Federal Highway Administration. "Distress Identification Manual for the long-term pavement performance program", Publication Year: June 2003. Online [Nov. 2012].

[13] U.S. Department of Transportation Federal Highway Administration. "Guidelines for Detection, Analysis, and Treatment of Materials-Related Distress in Concrete Pavements Vol II", Publication Number: FHWA-RD-01164, Date: March 2002. Online [Nov. 2012].
[14] U.S. Army Corps Of Engineers. "O\&M: PAVER Concrete Surface Airfields Pavement Condition Index (PCI)", UFC 3-270-05. 15 March 2001. Online [Nov. 2012].

[15] C. H. Higuera Sandoval, "Caracterización de la resistencia de la subrasante con la información del deflectómetro de impacto". Revista Facultad de Ingeniería UPTC, Vol. 19, No. 28. Pp. 73-92, 2010.

[16] H. A. Rondón \& F. A. Reyes. "Metodologías de diseño de pavimentos flexibles: Tendencias, alcances y limitaciones". Universidad Militar Nueva granada, Bogotá, Colombia, 2007 pp. 41-65.

[17] Consorcio troncal carrera 7. "Estudios y diseños troncal carrera 7, Informe final de pavimentos - parámetros de diseño", pp. 20, 2008.

[18] F. Sánchez. Caracterización del Tránsito Modulo 4". Online [Dic. 2012].

[19] Secretaría de Infraestructura Física, Municipio de Medellín. SOLTEC ING Soluciones Técnicas, "Ajuste y calibración de modelos de deterioro para el sistema de gestión vial e infraestructura asociada del Municipio de Medellín". Informe Final, pp. 1-47, junio 2011.

[20] ESRI. Understandingourworld, "Introducing ArcGIS 10.1". Online [Nov. 2012]. 